\title{
A Significant Approach for Graph Theory and Its Applications
}

\author{
K. Ramya ${ }^{1}, K$. Suganya ${ }^{2}$
}

${ }^{1}$ Assistant Professor, Department of Science and humanities, C. Abdul Hakeem College of Engineering and Technology, Vellore, Tamil Nadu, India

${ }^{2}$ Assistant Professor, Department of Computer Applications, Vivekananda College of Arts and Science, Sirkali, Tamil Nadu, India

\section{ABSTRACT}

Graph theory is a branch of discrete mathematics. Graph theory is the study of graphs which are mathematical structures used to model pair wise relations between objects. Now a days the role of graph theory in various filed is increasing, currently it provide greater functionality, combination, and low cost system into real world designed systems. Graph theory is in spot to play extensive roles in real application

Keywords : GPS, PERT

\section{INTRODUCTION}

A graph is an ordered pair $\mathrm{G}=(\mathrm{V}, \mathrm{E})$ consisting a set of vertices $\mathrm{V}$ with a set of edges $\mathrm{E}$. Graph theory is originated with the problem of Koinsber bridge, in 1735. This problem escort to the concept of Eulerian Graph. Euler studied the problem of Koinsberg Bridge and established a structure to resolve the problem called Eulerian graph. In 1840, A.F Mobius presented the idea of complete graph and bipartite graph and Kuratowski proved that they are planar by means of recreational problems. The concept of tree, (a connected graph without cycles was enacted by Gustav Kirchhoff in 1845, and he enrolled graph theoretical ideas in the calculation of currents in electrical networks or circuits. In 1852, Thomas Gutherie established the famous four color problem. Then in 1856, Thomas. P. Kirkman and William R.Hamilton measured cycles on polyhydra and contrived the concept called Hamiltonian graph by studying trips that visited certain sites exactly once [3]. In 1913, H.Dudeney mentioned a puzzle problem. Eventhough the four color problem was invented it was solved only after a century by Kenneth Appel and Wolfgang Haken. This is considered as origin of Graph Theory

\section{METHODS AND MATERIAL}

\section{A. Graph theory in Everyday Life}

There is n-number of applications of graph theory, few are represented as follows:

1. GPS or Google Maps: GPS or Google Maps are to find a shortest route from one destination to another. The destinations are Vertices and their connections are Edges consisting distance. The optimal route is determined by the software. Schools/ Colleges are also using this technique to pick up students from their stop to school. Each stop is a vertex and the route is an edge. A Hamiltonian path represents the efficiency of including every vertex in the route.

2.Traffic lights: The functioning of traffic lights i.e. turning Green/Red and timing between them. Here vertex coloring technique is utilized to solve conflicts of time and space by identifying the chromatic number for the number of cycles needed. 3.Social Networks We connect with friends via social media or a video gets viral, here user is a Vertex and other 
connected users create an edge therefore videos get viral when reached to certain connections.

4. To clear road blockage When roads of a city are blocked due to ice. Planning is needed to put salt on the roads. Then Euler paths or circuits are used to traverse the streets in the most efficient way.

5. While using Google to search for WebPages, Pages are linked to each other by hyperlinks. Each page is a vertex and the link between two pages is an edge.

6. The matching problem: In order to assign jobs to employees (servers) there is an analogue in software to maximize the efficiency.

\section{B. Scenarios of Graph Theory}

1. School Districts developing bus routes to pick up students to deliver to school. Each stop is a vertex and the route is an edge. A Hamiltonian path represents the efficiency of including every vertex in the route

2. Planning and processing the preparation of a meal. The use of PERT graphs to plan a course of action for projects

3. Shopping on Amazon or movies on Netflix. Relationship graphs are used to make recommendations for future shopping or films.

4.City planning to put salt on the roads when ice develops. Euler paths or circuits are used to traverse the streets in the most efficient way.

5. Visiting a zoo, water park or theme park and wanting to see certain attractions or devise an efficient route to see all of the attractions. A Hamiltonian path or circuit contains every vertex in the graph.

6. Examination on the spread of viruses/diseases. Vertex-edge graphs provide a visual for the network connection of those affected by the virus.

\section{Applications of Graph Theory}

Graph theory has its applications in diverse fields of engineering
- Electrical Engineering - The concepts of graph theory is used extensively in designing circuit connections. The types or organization of connections are named as topologies. Some examples for topologies are star, bridge, series, and parallel topologies.

- Computer Science - Graph theory is used for the study of algorithms. For example,

- Kruskal's Algorithm

- Prim's Algorithm

- Dijkstra's Algorithm

- Computer Network - The relationships among interconnected computers in the network follows the principles of graph theory.

- Science - The molecular structure and chemical structure of a substance, the DNA structure of an organism, etc., are represented by graphs.

- Linguistics - The parsing tree of a language and grammar of a language uses graphs.

- General- Routes between the cities can be represented using graphs. Depicting hierarchical ordered information such as family tree can be used as a special type of graph called tree.

\section{RESULTS AND DISCUSSION}

\section{A. Graph theory application for brain}

Graph theory applied to neuroimaging data can help us understand how the brain is organized. It may also help us understand the biologic underpinnings of behavioral function and dysfunction, particularly in patients with neurocognitive disorders, a population currently underserved by the radiologic community. A number of psychiatric and neurocognitive disorders can be classified as disconnection syndromes, in which there is damage to either white matter connections or association corticies bridging 
specialized sensorimotor regions $(\underline{8}-\underline{10})$. The emergence of particular symptoms can be theoretically related to particular types of damage to large-scale brain networks.

A number of studies in which researchers have used resting-state functional MR imaging and graph theoretical approaches have shown abnormalities in intrinsic brain networks in patients with different abnormal conditions, including Alzheimer disease (AD), schizophrenia, attention deficit hyperactivity disorder, epilepsy, and traumatic brain injury .For example, in patients with AD, Supekar et al found a significant decrease in the clustering coefficient and small-world properties in patients with $\mathrm{AD}$ compared with control subjects, consistent with lower regional connectivity and disruption of global organization of brain networks. These and other findings suggest that small-world metrics may be useful imaging-based biomarkers for a number of conditions. In addition, the robustness of a network to particular types of structural damage can be tested with lesion models. For example, He and colleagues examined the effect of random deletions of nodes and links versus targeted deletions of highly interconnected nodes and long-distance links in healthy subjects and those with $\mathrm{AD}$. In healthy subjects, the network was resistant to both types of attack; however, in patients with $\mathrm{AD}$, the network was approximately as robust to random failures but was particularly vulnerable to targeted attacks, presumably as a result of altered network organization (disrupted small-world architecture).

\section{B. Graph theory supporting languages}

Various computer languages are used to support the graph theory concepts. The main goal of such languages is to enable the user to formulate operations on graphs in a compact and natural manner
Some graph theoretic languages are

1. SPANTREE - To find a spanning tree in the given graph.

2. GTPL - Graph Theoretic Language

3. GASP - Graph Algorithm Software Package

4. HINT - Extension of LISP

5. GRASPE - Extension of LISP

6. IGTS - Extension of FORTRAN

7. GEA - Graphic Extended ALGOL (Extension of ALGOL)

8. AMBIT - To manipulate digraphs

9. GIRL - Graph Information Retrieval

Language

10. FGRAAL - FORTRAN Extended Graph

Algorithmic Language

\section{IV.CONCLUSION}

The graph theory has a wide application in various field of science and engineering course especially in electrical and computer engineering and also in communication industry. In graph theory approach is a useful tool for studying biological networks at a system level and provides insights on topology properties usage

\section{REFERENCES}

[1]. Mark Weiser, The Computer for the 21st Century, Scientific American, September 1991

[2]. M.Satyanarayanan, PervasiveComputing:Vision and Challenges, IEEE Personal Communications ,2001.

[3]. LalanaKagal,TimFinin, and AnupamJoshi,TrustBased Security inPervasive Computing Environments, Computer Communications, Elseiver, December 2001

[4]. Michael Collins,Simon Dobson, Paddy Nixon, S ecurity Issues with Pervasive Computing Frameworks, in workshop on privacy,Trust and 
Identity issues for Ambient Intelligence at Pervasive 2006 , pp 1-7, (2006)

[5]. Munirul, Haque and Sheikh Iqbal Ahamed, Security in Pervasive Computing: Current Status and Open Issues, International Journalof Network Security, Vol.3, No.3, PP.203-214, Nov.2006

[6]. Zeeshan Bilal, Ashraf Masood, Firdous Kausar, Security Analysis of Ultra-lightweight Cryptographic Protocol for Low-cost RFIDTags:Gossamer Protocol, in Proceedings of International Conference on Network-Based Information Systems, August 2009

[7]. Thomas Eisenbarth, Sandeep Kumar, Christof Paar and Axel Poschmann, Leif Uhsadel, A Survey of Lightweight-Cryptography Implementations, IEEE Design \& Test of Computers, November- December 2007

[8]. Jianhua Ma, Qiang fu Zhao, Vipin Chaudhary, Jingde Cheng, Laurence T. Yang, Runhe Huang, and Qun Jin, Ubisafe computing Vision and challenges, L.T.Yang et al.(Eds.): ATC 2006, LNCS4158, pp.386-397, Springer-Verlag Berlin Heidelberg 